\title{
SDR-Based High-Definition Video Transmission for Biomedical Engineering
}

\author{
Type of article: Conference abstract
}

\author{
Reinaldo P. Franca ${ }^{1}$, Ana C. B. Monteiro ${ }^{1}$, Vania V. Estrela ${ }^{2}$, Abdeldjalil Khelassi ${ }^{3}$, Y. Iano ${ }^{2}$, Navid \\ Razmjooy $^{4}$, Maria A. de Jesus ${ }^{2}$, Edwiges G. H. Grata ${ }^{2}$ \\ ${ }^{1}$ FEEC, UNICAMP, Campinas, SP, Brazil \\ ${ }^{2}$ Dep. of Telecommunications, Fluminense Federal University (UFF), RJ, Brazil \\ ${ }^{3}$ University of Tlemcen, Tlemcen, Algeria \\ ${ }^{4}$ Dep. of Electrical Engineering, Tafresh University, Tafresh, Iran \\ Corresponding Author: vania.estrela.phd@ieee.org
}

\begin{abstract}
:
Background: Software-Defined Radio (SDR) frameworks from cellular telephone base stations, e.g., Multiservice Distributed Access System (MDAS) and small cells, employ extensively integrated RF agile transceivers. The Internet of Medical Things (IoMT) is the collection of medical devices and applications that connect to healthcare IT systems through online computer networks. Medical devices equipped with Wi-Fi allow M2M communication, which is the backbone of IoMT and associated devices linked to cloud platforms containing stored data to be analyzed. Examples of IoMT include remote patient monitoring of people with chronic or long-term conditions, tracking patient medication orders and the location of patients admitted to hospitals, and patients' wearables to send info to caregivers. Infusion pumps connected to dashboards and hospital beds rigged with sensors measuring patients' vital signs are medical devices that can be converted to or deployed as IoMT technology.

Methods: This work proposes an SDR architecture to allow wireless High-Definition (HD) video broadcast for biomedical applications. This text examines a Wideband Wireless Video (WWV) signal chain implementation using the transceivers, the data transmitted volume, the matching occupied RF bandwidth, the communication distance, the transmitter's power, and the implementation of the PHY layer as Orthogonal Frequency Division Multiplexing (OFDM) with test results to evade RF interference.
\end{abstract}

Results: As the IoMT grows, the amount of possible IoMT uses increases. Many mobile devices employ Near Field Communication (NFC) Radio Frequency Identification (RFID) tags allowing them to share data with IT systems. RFID tags in medical equipment and supplies allow hospital staff can remain aware of the quantities they have in stock. The practice of using IoMT devices to observe patients in their homes remotely is also known as telemedicine. This kind of treatment spares patients from traveling to healthcare facilities whenever they have a medical question or change in their condition.

Conclusion: An SDR-based HD biomedical video transmission is proposed, with its benefits and disadvantages for biomedical WWV are discussed. The security of IoMT sensitive data is a developing concern for healthcare providers.

Keywords: Software-Defined Radio, Video Transmission, Biomedical Images, Telemedicine.

\section{Conflict of interest statement}

This article is a conference abstract presented at the International Congress on Health Sciences and Medical Technologies, Tlemcen Algeria 5-7 December 2019, ICHSMT'19. 
Medical Technologies Journal, Volume: 4, Issue: 3, July-September 2020, Pages:584-584. Doi :

https://doi.org/10.26415/2572-004X-vol4iss3p585-585

\section{Authors' biography}

No Biography.

\section{References}

No references 